\title{
On the resonant state of magnetization in array of interacting nanodots
}

\author{
P.D. Kimª, V.A. Orlov ${ }^{\mathrm{a}, \mathrm{b}, \mathrm{c}, *}$, R.Yu. Rudenko ${ }^{\mathrm{a}, \mathrm{b}}$, V.S. Prokopenko ${ }^{\mathrm{d}}$, I.N. Orlova ${ }^{\mathrm{d}}$, A.V.Kobyakoveva \\ ${ }^{a}$ Kirensky Institute of Physics Federal Research Center KSC Siberian Branch Russian Academy of Sciences 660036, Krasnoyarsk, Russia \\ ${ }^{b}$ Siberian Federal University, 660041, Krasnoyarsk, Russia \\ ${ }^{c}$ Federal Research Center KSC Siberian Branch Russian Academy of Sciences 660036, Krasnoyarsk, Russia \\ ${ }^{d}$ Krasnoyarsk State Pedagogical University after V.P. Astaf'ev, 660049, Krasnoyarsk, Russia
}

\begin{abstract}
Development of the interpretation of the phenomenon of the lift of the magnetic resonance frequencies degeneracy caused by the magnetostatic interaction in assemblies of nanodisks has been done. The difference of the resonance behavior of magnetic vortexes in a round and rectangular nanodots has been studied experimentally and explained.
\end{abstract}

Keywords: nanodots, magnetization, dispersion law, Tiel's equation

PACS: 75.75.Jn, 75.78.-n

\section{Introduction}

It is known, at certain ratios between the thickness and width (diameter) of nanodots, the stable distribution of the magnetization occurs in the form of a vortex with the Bloch point in the center of a magnet. The theory for describing the dynamics of the magnetization was developed on the basis of the Thiel equation (in terms of collective variables [1]-[6]). It was shown that the core of magnetic vortex executes gyrotropic motion around the axis of a nanodisk similar to the Larmor motion of a charged particle around the magnetic field lines.

The experimental tools for studying nanodots are also widely developed (e.g., in [7, 8]). The cited works present the results of experimental studies of the magnetic properties of the individual nanodots. However, most experiments deal with films, i.e. arrays of fairly separated nanodots. For this reason, the interaction between the magnetic subsystems of elements of an array is usually disregarded [9]. At the same time, the long-term magnetostatic interaction can affect at least collective modes of rotational motion of the core if not the static or quasistatic characteristics of magnetization [12].

We would like also mention the works, where the magnetostatic interaction in assemblies of magnetic nanoparticles was studied $[13,14]$, and the problem of the oscillatory modes in a pair of coaxial magnetostatically interacting nanodisks was analytically solved [15].

\footnotetext{
${ }^{*}$ Corresponding author

Email address: orlhome@rambler.ru (V.A. Orlov)
}

\section{Experimental equipment and samples}

The arrays of circular and square nanodots for our studies were formed by the "lift-off" technique from a continuous film prepared with thermal sputtering from an 80HXC alloy on silicon substrate [16].

The morphology and the magnetic relief of the films surface were studied with a the "Veeco MultiMode NanoScope IIIa SPM System". The chirality direction $q= \pm 1$ was determined from the distribution of the gradient of the magnetic forces acting on the probe of the cantilever. Diameter and thickness of the particles were 3 microns and $100 \mathrm{~nm}$, correspondingly.

The magnetic resonance properties of the nanodot array were studied with the ferromagnetic resonance (FMR) spectrometer operating at the frequency of 373 $\mathrm{MHz}$ and $415 \mathrm{MHz}$. The sample was placed in the antinode of a magnetic field of the cavity, which was of a short-circuited quarter-wave waveguide with a 4-mm wide central strip. The differential absorption curves were obtained for the sample in dependence of the base field (Fig. 1). The absorption curves in Fig. 1 have the form of superposition of curves with different absorption widths and resonance frequencies. This means the splitting of the resonance frequency of the gyrotropic motion of vortices.

\section{Magnetostatic interacting nanodisks}

Below, we consider the model situation that will help us qualitatively understand the origin of the lift of the degeneracy of the resonance frequency. Here we present a refinement of the calculations from works [16] for 2Darray of nanodots. Let us consider a square $2 \mathrm{D}$ array of 


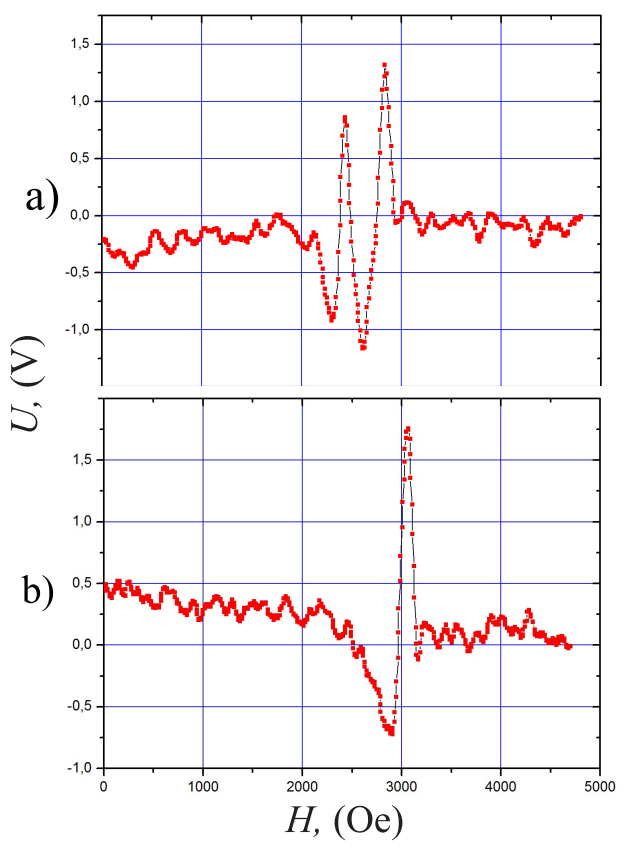

Figure 1: Differential absorption curves obtained in the FMR experiment with the circular nanodots for arrais with the distance between the disks 2 microns (a), and 4 microns (b).

cylindrical nanodots with the centers separated by equal distance $d$.

It is known, one of the equilibrium magnetization distributions in circular nanocylinders is the vortex structure[17]-[20]. Below, we use an approximation in which the profile of the magnetization of the vortex changes rigidly at the displacement of the core from the center (rigid magnetic vortex model). The behavior of the core is described by the equation of motion in the form

$$
\mathbf{G} \times \mathbf{v}-\nabla U+\mathbf{F}=0 .
$$

Here, $\mathbf{G}$ is the gyrovector, $\mathbf{v}$ is velocity of the core the magnetic vortex, $U$ is the potential energy of the magnetization (its variation at the displacement of the core from the disk center is attributed, as a rule, to an increase in the magnetostatic energy). According to (1) if $\mathbf{F}=0$, the vortex core involves in the complex motion with the gyroforce [21]-[26]. We added this equation with the force $\mathbf{F}$ caused by the magnetostatic interaction between array elements.

Consider the mechanism of the magnetostatic interaction by the example of two neighboring disks. As was shown in [27], the magnetic field configuration beyond the nanodot is similar to the configuration of the field of a magnetic dipole with the satisfactor accuracy. Therefore, below we can use the dipole approximation to estimate the energy of interaction between cylinders. In [28], the collective modes were thoroughly analytically calculated using the model where only the magnetizations of identically oriented cores interact.

We approximate the interaction between nanodisks by parabolic potential well $\nabla U=\kappa \mathbf{r}$, where $\kappa$ is the socalled effective rigidity of a magnetic subsystem. In this case, in the projections onto the system of coordinates, equations for $n$-th disk is

$$
\begin{gathered}
-G \dot{y}_{n}-\kappa x_{n}+F_{x n}=0, \\
G \dot{x}_{n}-\kappa y_{n}+F_{y_{n}}=0 .
\end{gathered}
$$

Let us estimate the force $F$. In the dipole approximation, the energy of magnetostatic interaction between disks distant from each other by $i$ periods along the $x$ axis and by $j$ periods along the $y$ axis can be presented as $W_{n, m, i, j}=$ $\left(\mathbf{M}_{n, m} \mathbf{M}_{n+i, m+j}-3\left(\mathbf{M}_{n, m} \mathbf{r}_{i, j}\right)\left(\mathbf{M}_{n+i, m+j} \mathbf{r}_{i, j}\right) /\left(i^{2}+j^{2}\right) d^{2}\right) /$ $\left(i^{2}+j^{2}\right)^{3 / 2} d^{3}$. Here, $\mathbf{r}_{i, j}$ is the radius vector connecting centers of nanodisks. We will seek for the solution of system (2) in the form

$$
\begin{aligned}
& x_{n, m}=a_{n, m} \cos \left(k_{x} X_{n, m}+k_{y} Y_{n, m}-q_{n, m} p_{n, m} \omega t\right), \\
& y_{n, m}=b_{n, m} \sin \left(k_{x} X_{n, m}+k_{y} Y_{n, m}-q_{n, m} p_{n, m} \omega t\right) .
\end{aligned}
$$

Here, the parameter $q_{n, m}= \pm 1, p_{n, m}= \pm 1$ is the chirality and polarity of the vortex with the number $(n, m)$, $X_{n, m}, Y_{n, m}$ are the nanodots center coordinates, and $k_{x}, k_{y}$ are the projections of the wave number onto the coordinate axes. Now, let us consider the case when one array contains disks with only two possible implementations $\left((q, p)\left(q^{\prime}, p^{\prime}\right)\right)$, which are arranged in the staggered order.

Although the chosen model is somewhat artificial, it allows us to qualitatively explain the phenomenon of the lift of the degeneracy of the resonance frequency. Substituting the probe solutions to Eq. (2) for disks of two different kinds, we obtain biquadratic equation for the unknown $\omega$. The solution for the modes of resonance motions

$$
\omega^{2}=\frac{B}{2 A} \pm \sqrt{\left(\frac{B}{2 A}\right)^{2}-\frac{C}{A}},
$$

where

$$
\begin{aligned}
& A=G^{2} G^{2} \\
& B=\left(G^{2}+G^{2}\right)\left(\kappa+\varepsilon S_{o}^{(1)}\right)\left(\kappa+\varepsilon S_{o}^{(2)}\right)+ \\
& 2 G G^{\prime} \varepsilon^{2} S_{e}^{(1)} S_{e}^{(2)} \\
& C=\left(\left(\kappa+\varepsilon S_{o}^{(1)}\right)^{2}-\varepsilon^{2} S_{e}^{(1)^{2}}\right)\left(\left(\kappa+\varepsilon S_{o}^{(2)}\right)^{2}-\varepsilon^{2} S_{e}^{(2)^{2}}\right) .
\end{aligned}
$$


Here, we introduced the designations

$$
\begin{aligned}
& S_{e}^{(1)}=\sum_{i+j=1,3,5 \ldots}\left(\frac{i^{2}-2 j^{2}}{\left(i^{2}+j^{2}\right)^{5 / 2}} \cos \left(k_{x} d i\right) \cos \left(k_{y} d j\right)\right), \\
& S_{o}^{(1)}=\sum_{i+j=2,4,6 \ldots}\left(\frac{i^{2}-2 j^{2}}{\left(i^{2}+j^{2}\right)^{5 / 2}} \cos \left(k_{x} d i\right) \cos \left(k_{y} d j\right)\right), \\
& S_{e}^{(2)}=\sum_{i+j=1,3,5 \ldots}\left(\frac{j^{2}-2 i^{2}}{\left(i^{2}+j^{2}\right)^{5 / 2}} \cos \left(k_{x} d i\right) \cos \left(k_{y} d j\right)\right), \\
& S_{o}^{(2)}=\sum_{i+j=2,4,6 \ldots}\left(\frac{j^{2}-2 i^{2}}{\left(i^{2}+j^{2}\right)^{5 / 2}} \cos \left(k_{x} d i\right) \cos \left(k_{y} d j\right)\right), \\
& \varepsilon=\frac{\mu_{0} M^{2}}{\pi R_{0}^{2} d^{3}} .
\end{aligned}
$$

Parameters $G, G^{\prime}$, and $\kappa$ are taken from the works $[2,8,26]$ :

$$
\begin{aligned}
& G=q p \frac{2 \pi M_{S} L}{\gamma}(1-p h), \kappa=\frac{40}{9} \frac{\pi M_{S}^{2} L^{2}}{R}\left(1-h^{2}\right) . \\
& G^{\prime}=q^{\prime} p^{\prime} \frac{2 \pi M_{S} L}{\gamma}\left(1-p^{\prime} h\right),
\end{aligned}
$$

Here, $L$ is the nanodisk thickness and $\gamma$ is the gyromagnetic ratio. The dimensionless field $h=H / H_{S}$ is applied perpendicular to the disk plane.

In the limit case $d \gg R$, when disks do not interact, Eq. (4) yields the well-known result for the core gyrotropic motion frequency in the solitary disk: $\Omega_{0}=\kappa / G \quad \Omega_{0}^{\prime}=\kappa / G^{\prime}$. The presence of a multiplet in the FMR absorption curve in zero external field was predicted in [29] and confirmed in part in the experiment [27], were a system of two or four interacting disks.

Figure 1 shows differential absorption curves obtained by us in the FMR experiment. It is seen that practically one resonance curve is observed, when the distance between discs is 4 microns (a). The splitting of this curve onto several resonance peaks occur, when the distance between the discs became 2 microns (b). We interpret this splitting as the lift of the degeneracy of the resonance frequency. The maximum splitting calculated using formula (4) in the long-wavelength limit is about $\Delta h \approx 0.2$. This is in satisfactory agreement with the experimental data presented in Fig.1a.

The theory of the shape of the splitting resonance curve is not developed yet. That is why we describe the experimental data illustrated in Fig. 1, introducing a fitted formula. Assume the absorbed power to be determined by characteristic the sum of the independent Lorentz resonance curves

$$
P(\omega, h) \sim \sum_{i} \frac{\rho_{i} \omega \Gamma(h)}{\left(\omega^{2}-\omega_{i}(h)^{2}\right)^{2}+4 \omega^{2} \Gamma(h)^{2}} .
$$

Here $\rho_{i}$ is a weighting coefficient and $\omega$ is a frequency. We used the dissipation coefficient in the form [2]: $\Gamma(h)=\kappa D /\left(G^{2}+D^{2}\right) . \quad D$ is the coefficient of quasiviscous friction of the magnetic vortex core [2]. The total absorbed power at the fixed frequency of the experimental facility $\omega$ can be considered as a sum of terms with different $\omega_{i}(h)$ corresponding to different polarity and chirality combinations

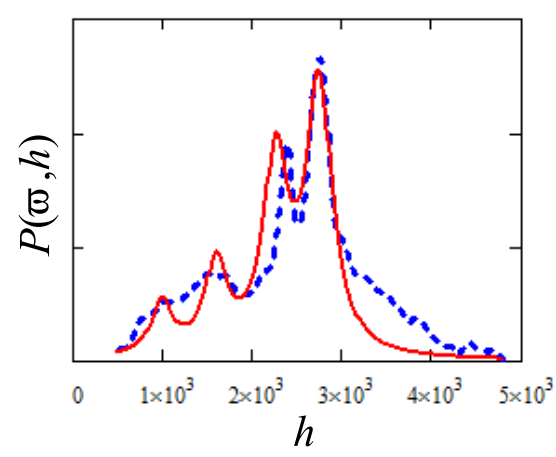

Figure 2: Integral resonance curves for circular nanodot arrays obtained experimentally (dashed line) and calculated using the formula (8) (solid line)

Figure 2 shows the comparison of the integral absorption curve obtained from the experimental data corresponding to Fig. 1a and the phenomenological curve obtained using expression (8) in the long-wavelength limit $\left(k_{x}, k_{y} \rightarrow 0\right)$. In this formula, the sum of four terms corresponding to the frequencies of the modes of the formula (4). The weighting coefficients $\rho_{i}$ we choose so that a phenomenological curve of best fit of the experimental curve.

\section{Circular and square nanospots}

We carred out the experimental study of an array of square nanodots with the following characteristics. The size of the nanoparticles is $3 \times 3$ microns the thickness is $50 \mathrm{~nm}$, and the distance between particles is 3 microns. Figure 3 shows the effect of the parallel component of the field $\left(H_{\|}\right)$on the perpendicular resonance field $\left(H_{\perp}\right)$. The results of the experiment are shown in Figure 3.

We believe that the enhanced sensitivity of the resonance frequency of the magnetization in a square magnet is related to the shape of the effective potential well where the vortex core is located as a quasi-particle. In a circular nanomagnet, the well has the form of a paraboloid of revolution; therefore, the gyrotropic frequency of the core is almost independent of the radius of its revolution around the disk center and, consequently, on amplitudes of the applied ac and dc magnetic fields.

The situation is different in square nanomagnets. Let us consider a simplified model of the rigid magnetic core. The displacement of the vortex core induces charges with a density $\sigma$ at the lateral surface, which interact with each other. Let us calculate the energy of their pair interaction. For the total energy of interaction between side surface of a square, we can write $E=\frac{\mu_{0}}{4 \pi} \iint \sigma_{1} \sigma_{2} d s_{1} d s_{2} /\left|\mathbf{r}_{1}-\mathbf{r}_{2}\right|$. The integration is performed on the surface area of the lateral nanodots, 


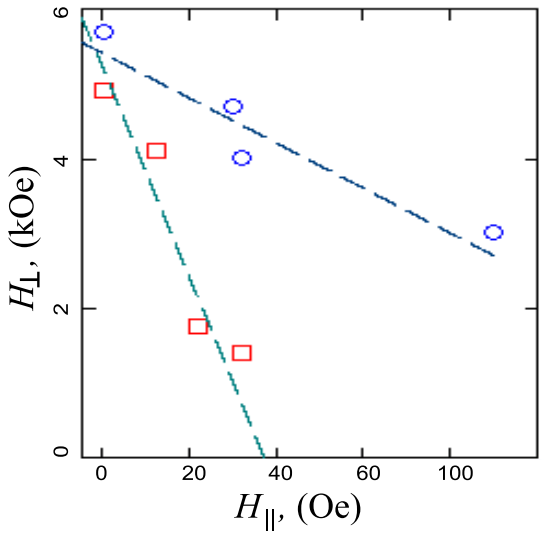

Figure 3: The perpendicular resonance field vs the planar field for arrays of the round nanodots (circle) and the square nanodots (squares).

as it has been done in the work [10] for the circular nanodots. The results of the numerical integration in the region of small displacements of the core $(<a / 4$, where $a$ is the length of the square) is approximated well by the function $W_{b}=5 \frac{\mu_{0} M_{S}^{2} L^{2} a}{\pi}\left(\left(\frac{x}{a}\right)^{2}-\frac{1}{4}\right)\left(\left(\frac{y}{a}\right)^{2}-\frac{1}{4}\right)$.

In the planar dc magnetic field, the vortex core shifts from the center and appears in the potential region with the smaller quasi-rigidity coefficient. This reduces the velocity of the core and frequency of its revolution. This also explains the high sensitivity of the resonance curves to the orientation of the sample relative to the direction of the planar magnetic field. In the circular nanodots, this effect is much weaker, since the effective rigidity of the magnetic subsystem in circular magnets is nearly independent of the core displacement.

\section{Conclusion}

The phenomenon of lift of the degeneracy of the resonance frequency is due to magnetostatic interaction nanodots. Disks with a combination of different polarities and chiral drives provide various magnetization oscillations modes.

The square nanodots revealed a strong dependence of the resonance frequency of the planar magnetic field. The round disks, this dependence is weaker. We believe that the reason for this difference in the profile of the potential well of parabolic in square nanodots.

\section{Acknowledgments}

Authors are grateful to V. Ya. Prints for help in preparing dot arrays. Sections 3 and 4 of the work was supported by the Russian Science Foundation, project no. 14-15-00805. Section 2 of the work was supported
Russian Foundation for Basic Research (RFBR) project no. 16-32-00103.

\section{References}

[1] A.A. Thiel, Phys. Rev. Lett. 30, (1975) 230

[2] J. Kim and S.-B. Choe, J. of Magn. 12, (2007) 113

[3] F.G. Mertens, H.J. Schnitzer and A.R. Bishop, arXiv:condmat/9902151v1 10 Feb (1999)

[4] F.G. Mertens and A.R. Bishop, arXiv:cond-mat/9903037v1 2 Mar (1999)

[5] B. A. Ivanov, D. D. Sheka, Pisma v Zh. Eksp. Teor. Fiz., 82, (2005) 489, [JETP Letters, 82 (2005) 436]

[6] B. A. Ivanov, G. G. Avanesyan, A. V. Khvalkovskiy, N. E. Kulagin, C. E. Zaspel, K. A. Zvezdin, Pisma v Zh. Eksp. Teor. Fiz., 91, (2010) 190, [JETP Letters, 91 (2010) 178].

[7] B. Pigeau, G. de Loubens, O. Klein, A. Riegler, F. Lochner, G. Schmidt, L. W. Molenkamp, V. S. Tiberkevich and A. N. Slavin, Appl. Phys. Lett. 96 (2010) 132506

[8] G. Loubens, A. Riegler, B.Pigeau, F. Lochner, F. Boust, K.Y. Guslienko, H. Hurdequint, L.W. Molekamp, G. Schmidt, A.N. Slavin, S. Tiberkevich, N. Vukadinovic and O. Klein, Phys. Rev. Lett. B 102 (2009) 177602

[9] V. Novosad,F.Y. Fradin, P.E. Roy, K.S. Buchanan, K.Yu. Guslienko and S.D. Bader, Phys. Rev. B 72 (2005) 024455

[10] P. D. Kim, V. A. Orlov, V. S. Prokopenko, S. S. Zamai, V. Ya. Prints, R. Yu. Rudenko, T. V. Rudenko, Fizika tverdogo tela, 57 (2015) 29, [Physics of the Solid State, 57 (2015) 30].

[11] Yu. P. Ivanov, E. V. Pustovalov, A. V. Ognev, L. A. Chebotkevich, Fizika tverdogo tela, 51 (2009) 2167, [Physics of the Solid State, 512300 (2009)].

[12] K.Yu. Guslienko, V. Novosad, Y. Otani, H. Shima and K. Fukamichi, Phys. Rev. B 65, 024414 (2001)

[13] M. Beleggia, S. Tandon, Y. Zhu, M.De. Graef, JMMMM 278, 270 (2004)

[14] V.A. Ignatchenko, I.S. Edelman, D.A. Petrov, Phys. Rev. B 81, 054419 (2010)

[15] S.S. Cherepov, B.C. Koop, V. Korenivski, D.C. Worledge, A.Yu. Galkin, R.S. Khymyn and B.A. Ivanov, Phys. Rev. Lett. 109, 097204 (2012)

[16] P.D. Kim, V.A. Orlov, R.Yu. Rudenko, V.S. Prokopenko, I.N. Orlova, S.S. Zamai Pisma v Zh. Eksp. Teor. Fiz., 101 (2015) 620, [JETP Letters, 101 (2015) 562].

[17] J. Miltat and A.Thiaville, Science 298, 555 (2002)

[18] A. Puzic, B. Van Waeyenberge, K. W. Chou, P. Fischer, H. Stoll, G. Schutz, T. Tyliszczak, K. Rott, H. Bruckl, G. Reiss, I. Neudecker, Th. Haug, M. Buess and C. H. Back, J. of Appl. Phys. 97, 10 E704 (2005)

[19] T. Shinjo, T. Okuno, R. Hassdorf, K. Shigeto and T. Ono, Science, 289, 930 (2000)

[20] J. Raabe, R. Pulwey, R. Sattler, T. Schweinbock, J. Zweck and D. Weiss, J. of Appl. Phys. 88, 4437 (2000)

[21] O. Klein, G. de Loubens, V. V. Naletov, F. Boust, T. Guillet, H. Hurdequint, A. Leksikov, A. N. Slavin, V. S. Tiberkevich and N. Vukadinovic, Phys. Rev. B 78, 144410 (2008)

[22] K.-S. Lee and S.-K. Kim, Appl. Phys. Lett. 91, 132511 (2007)

[23] V.P. Kravchuk, Yu. Gaididei and D.D. Sheka, Phys. Rev. B 80, 100405(R) (2009)

[24] K.-S. Lee, K.Yu. Guslienko, J.-Y. Lee and S.-K. Kim, Phys. Rev. B 76, 174410 (2007)

[25] B.A. Ivanov and G.M. Wysin, Phys. Rev. B 65, 134434 (2002)

[26] K.Yu. Guslienko, B.A. Ivanov, V. Novosad, Y. Otani, H. Shima and K. Fukamichi, J. of Appl. Phys. 91, 8037 (2002)

[27] A. Vogel, A. Drews, T. Kamionka, M. Bolte and G. Meier, Phys. Rev. Lett 105, 037201 (2010)

[28] A. Yu. Galkin, B. A. Ivanov, Zh. Eksp. Teor. Fiz., 136 (2009) 87, [JETP, 109 (2009) 74].

[29] J. Shibata, K. Shigeto and Y. Otani, Phys. Rev. B 67, 224404 (2003) 